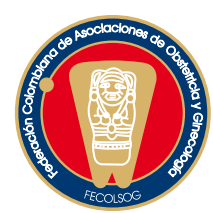

\title{
VARIACIONES MORFOLÓGICAS EN LA SÍNFISIS PÚBICA HUMANA. SERIE DE CASOS
}

\section{A case-series of morphological variations in human symphysis pubis}

Jorge Eduardo Duque-Parra. B.Sc., M.Sc., Ph.D.*, John Barco-Ríos, M.D., B.Sc.**

Recibido: enero 27/10 - Aceptado: agosto 9/10

\section{RESUMEN}

Objetivo: estudiar la cara posterior del disco sinfisiario púbico en cadáveres humanos adultos con el propósito de hallar posibles variantes anatómicas.

Metodología: se disecó la región de la articulación interpúbica y se evaluaron las características anatómicas. Se estudiaron cincuenta y tres sínfisis púbicas (4 femeninas y 49 masculinas) mediante abordaje anatómico, incidiendo la pared abdominopélvica con el fin de despejar los elementos asociados al pubis.

Resultados: el examen macroscópico de la sínfisis púbica mostró un aspecto uniforme y liso en su cara posterior, excepto en dos cadáveres de sexo masculino. Uno de ellos presentaba dos protuberancias nodulares contiguas de distinto tamaño y alineadas verticalmente sobre la cara posterior del disco sinfisiario, mientras que otro cadáver presentó un reborde, a manera de cresta, orientado también verticalmente sobre la cara posterior de dicho disco. Las dos protuberancias halladas presentaron comunicación directa con el interior del disco sinfisiario, a manera de

* Programa de Medicina, Departamento de Ciencias Básicas, Universidad de Caldas. Departamento de Ciencias Básicas Biológicas, Universidad Autónoma, Manizales (Colombia). Correo electrónico: jduqueparra@ gmail.com

** Programa de Medicina, Departamento de Ciencias Básicas, Universidad de Caldas. Manizales (Colombia). ampulaciones, con líquido intraarticular derivado del esbozo de cavidad que posee el disco.

Conclusión: las nodulaciones y el reborde descritos podrían corresponder a alteraciones patológicas articulares como diastasis púbicas, pero sin separación de los cuerpos del pubis.

Palabras clave: pubis, sínfisis, anatomía macroscópica, cápsula sinovial, diastasis púbica.

\section{SUMMARY}

Objective: studying the posterior side of the pubic symphysis (joint containing a fibrocartilaginous disc) in adult human corpses to find possible anatomical variations.

Methodology: the posterior side of the symphyseal joint was dissected and its anatomical characteristics were evaluated. Fifty-three pubic symphyses (4 females and 49 males) were evaluated in an anatomic approach by making an incision in the abdominal-pelvic wall to reveal elements associated with the pubis.

Results: macroscopic examination of the pubic symphysis revealed uniform, smooth posterior faces, except in two male cadavers. One of them had two, different sized, contiguous nodular protuberances which were vertically aligned on the posterior face of the symphysis disc whilst the other cadaver presented a border, in the form of a crest, also vertically orientated on the posterior face of such disc. Both protuberances so found had direct communication within the symphysis disc, 
like ampoules, with intra-articular liquid derived from the outlined disc's cavity.

Conclusion: the nodulations and border described above could have corresponded to articular pathological alterations, such as pubic symphysis diastasis, but without being separated from the body of the pubis.

Key words: pubis, symphysis, gross anatomy, synovial capsule, pubis diastasis.

\section{INTRODUCCIÓN}

La palabra sínfisis proviene del griego "Symphysis", que significa 'crecimiento unido', donde la superficie de los huesos que se articulan están recubiertas por una capa muy fina de cartílago articular hialino, quedando en medio de ellas un disco grueso de fibrocartílago. ${ }^{1-3}$ Dicha articulación se caracteriza por ser de gran fortaleza ${ }^{1,4}$ pero de movilidad limitada, debido a la compresión en la parte fibrosa de la unión. ${ }^{1}$ La sínfisis púbica se encuentra conformada por las caras articulares de los dos huesos púbicos, las cuales están revestidas por cartílago hialino ${ }^{2,3,5,6,7}$ localizándose entre ellas un disco fibrocartilaginoso ${ }^{2,5,7,8}$ que aparece en asociación íntima con el tejido conectivo denso de los ligamentos articulares; de allí que el disco sea considerado como una forma de transición entre el cartílago y el tejido conectivo denso. ${ }^{4}$ Este fibrocartílago presenta una cara dorsal muy angosta y de superficie lisa, mientras que su cara anterior se ensancha notablemente y es entrecruzada por una capa de haces fibrosos que la conectan directamente con los tendones de los músculos rectos abdominales. ${ }^{5}$

Al fibrocartílago articular púbico se le conoce también con el nombre de ligamento interóseo, el cual presenta forma de cuña como se mencionó anteriormente, donde su base está dirigida hacia el periné y su vértice hacia la excavación pélvica; lateralmente se relaciona con las carillas articulares de los huesos púbicos que lo cubren por completo. Su periferia es dura, densa y resistente, mientras que su porción central es más blanda. El fibrocartílago suele ser más ancho y grueso en la mujer, pero con una mayor altura en el hombre. ${ }^{2}$

A diferencia de los discos intervertebrales, en el centro del disco fibrocartilaginoso del pubis suele haber una pequeña cavidad, con orientación longitudinal y llena de líquido. ${ }^{1,56}$ Esta es de mayor dimensión en la mujer, ${ }^{2,5,8}$ pero carece, en términos generales, de las especificaciones de una articulación sinovial o diartrósica. ${ }^{4}$ Es por ello que entre las anfiartrosis o articulaciones sin movimiento aparente se cuenta la sínfisis del pubis. ${ }^{2,8,9}$ Sin embargo, debe tenerse en cuenta que la presencia de una cavidad central en el disco muestra las características de una diartrosis intermedia, la cual se clasifica mejor como una diartroanfiartrosis. ${ }^{9}$ La cavidad del disco fibrocartilaginoso es muy amplia, de tal manera que entra en contacto con los cuatro ligamentos periféricos de esta articulación; además, esta cavidad está recubierta internamente de una verdadera cápsula sinovial rudimentaria, que se clasifica entonces dentro de las diartrosis monoaxiles, que son variantes poco comunes y sin características completas de diartrosis. Cuando el disco no presenta cavidad entonces se clasifica como una anfiartrosis. ${ }^{9}$ Se ha encontrado que la cavidad del fibrocartílago aparece generalmente después de la infancia, pero sin revestimiento sinovial. ${ }^{3}$

Los ligamentos de la sínfisis del pubis se relajan por acción de una hormona no esteroidea llamada relaxina, ${ }^{8,10,11}$ que es secretada por el cuerpo lúteo del ovario durante la gestación. ${ }^{8,10,12}$ Esta hormona peptídica, además de actuar sobre el tejido conectivo, lo hace también sobre la musculatura lisa del cuello del útero, facilitando la dilatación ${ }^{1,2,8,12}$ para el pasaje del feto a través del canal del parto. ${ }^{1,2,8,12,13}$ Se estima que la separación de la sínfisis púbica durante el trabajo de parto tiene una incidencia de 1 en 300 a 1 en 30.000 embarazos, además, en casos traumáticos se ha presentado disrupción sinfisiaria hasta de $6,2 \mathrm{~cm},{ }^{14}$ lo cual provoca ensanchamiento y pérdida de la solidez de dicha articulación, que se manifiesta con cierto grado de inestabilidad sinfisiaria. ${ }^{15}$ 
Debido a que la pelvis del infante es más flexible y, por tanto, más dúctil que la del adulto por sus componentes cartilaginosos —entre ellos los de la sínfisis del pubis - son raras las fracturas; no obstante, la separación traumática completa de la sínfisis del pubis puede ser más frecuente en los infantes. ${ }^{16}$

Se debe tener un conocimiento anatómico exacto de la sínfisis púbica y los tejidos blandos que la circundan ${ }^{17}$ para poder inferir sobre las posibles alteraciones que sufre esta articulación cuando múltiples factores inciden sobre ella, como la patogénesis asociada con el dolor de la región inguinal, que es una queja común de los atletas que participan en distintos deportes. Por lo tanto, es importante diagnosticar tempranamente cualquier lesión sobre esta zona y tratarla con prontitud, pues de no controlarse a tiempo, se puede convertir en una lesión crónica de difícil tratamiento.

Se plantea la necesidad de investigar aquellos detalles morfológicos que puedan salirse de la normalidad aparente, los que no se encuentran registrados en los textos especializados (variantes anatómicas), e inferir posibles alteraciones patológicas o rasgos adaptativos del organismo frente al estrés mecánico, basándose en observaciones empíricas.

Por lo tanto, el objetivo de la presente investigación, fue estudiar la estructura de la cara posterior del disco sinfisiario en humanos adultos, con el propósito de hallar posibles variantes anatómicas.

\section{METODOLOGÍA}

Una serie de cincuenta y tres pelvis de cadáveres adultos (4 femeninas y 49 masculinas), fijadas con formalina (solución con formol al 10\%), fueron examinadas para determinar exclusivamente la uniformidad de la continuidad entre la cara posterior del disco sinfisiario y los componentes púbicos del hueso coxal, es decir, determinar si ésta era lisa o discontinua en el eje axial del disco sinfisiario púbico. Se excluyeron las pelvis que no estaban íntegras (sin disección previa) y no hubo valoración de ningún tipo de alteración clínica o quirúrgica, por lo que la escogencia fue aleatoria. Sólo se estudiaron los cadáveres adultos de dos salas de disección en dos universidades, que ingresaron a estas instituciones por un período de un año. A cada uno se le realizó un análisis descriptivo morfológico.

Del total de los pubis disecados, 13 habían sido previamente semidisecados como piezas individuales y los 40 restantes se disecaron sin separarlos de los cadáveres. La inspección de los componentes púbicos se valoró macroscópicamente, mientras que el abordaje anatómico se logró incidiendo la pared abdominal inferior para, después de ingresar a la región peritoneal, replegar los colgajos y poder inspeccionar el disco sinfisiario del pubis, previa desadherencia de la fascia prevesical.

\section{RESULTADOS}

Todas las pelvis estudiadas estaban en buen estado macroscópico para su disección, no presentaban alteraciones patológicas evidentes a la exploración superficial y pertenecían a personas adultas (de las que no se sabe su edad). En dos cadáveres masculinos se notó discontinuidad de la cara posterior del disco sinfisiario. Uno de los cadáveres reveló la existencia de dos protuberancias nodulares contiguas de distinto tamaño, alineadas verticalmente sobre la cara posterior del disco. La protuberancia mayor presentó un diámetro de $12 \mathrm{~mm}$ y una altura de $15 \mathrm{~mm}$, mientras que la protuberancia menor presentó un diámetro de $5 \mathrm{~mm}$ y una altura de $4 \mathrm{~mm}$ (figuras 1 y 2). Las dos protuberancias tenían una apariencia sacular, de consistencia blanda al tacto y con un contenido gelatinoso. Al abordar con un alfiler la protuberancia mayor se notó que había continuidad con la porción más profunda del disco sinfisiario, es decir con su cavidad (figura 3). En la protuberancia menor se hizo aún más evidente su aspecto nodoso y sacular, con una membrana delgada, y también se comprobó que había continuidad con la cavidad del disco sinfisiario. En el otro cadáver se encontró un reborde o cresta con orientación vertical y dispuesta, también sobre la cara posterior del 
Figura 1. Corte sagital de la sínfisis púbica mostrando dos nodulaciones. El alfiler está clavado sobre la nodulación menor.

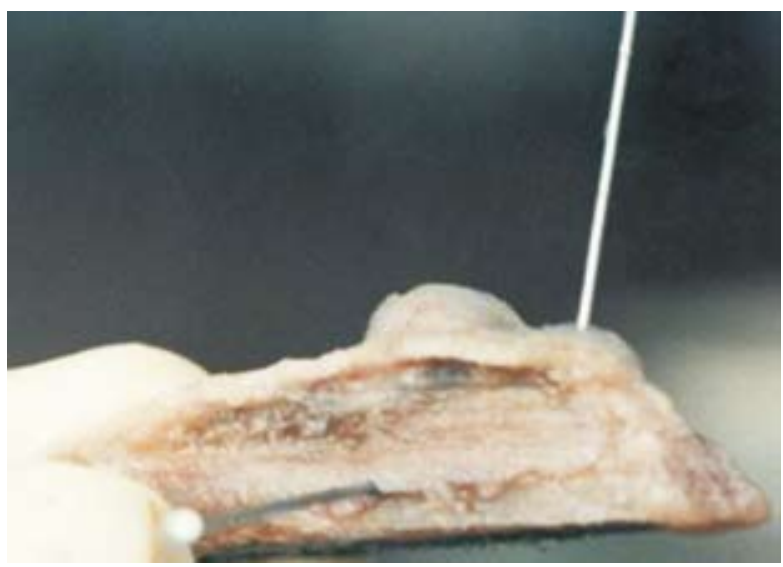

Figura 3. Corte sagital de la sínfisis púbica en el que se aprecia, por medio del alfiler, la comunicación que hay entre la nodulación mayor con la cavidad del disco sinfisiario.

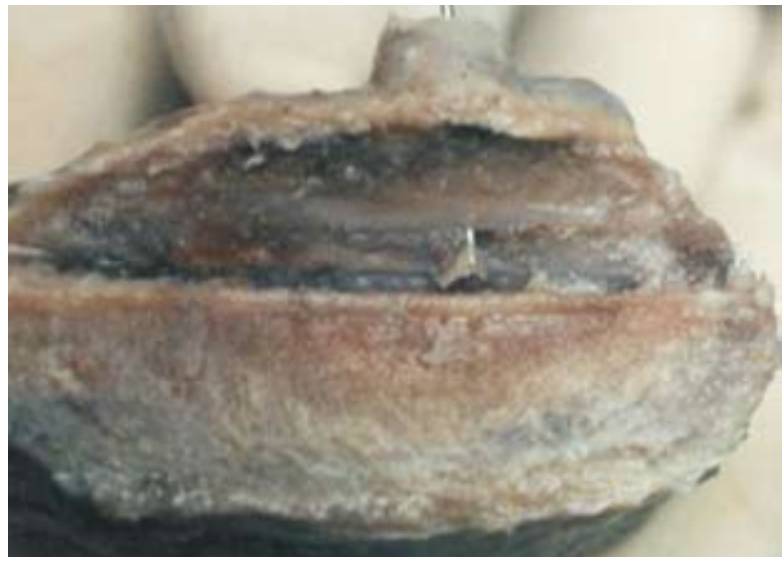

disco (figura 4). Estos dos casos contrastan notoriamente con la relación de continuidad y uniformidad que presentaron las sínfisis púbicas de los demás cadáveres examinados, los cuales coinciden con los conceptos de la anatomía macroscópica que describen esta parte del disco sin ningún tipo de elevamiento o protrusión.

\section{DISCUSIÓN}

De acuerdo con lo referido por Testeut y Latarjet, ${ }^{9}$ en el interior del disco sinfisiario se puede presentar una bolsa sinovial, que caracteriza esta sínfisis como una diartroanfiartrosis. Debido a la relación directa que se encontró entre el interior del disco y las dos
Figura 2. Vista horizontal de pelvis donde se aprecian las dos nodulaciones sobre la cara posterior de la sínfisis púbica. El alfiler está clavado sobre la nodulación mayor.

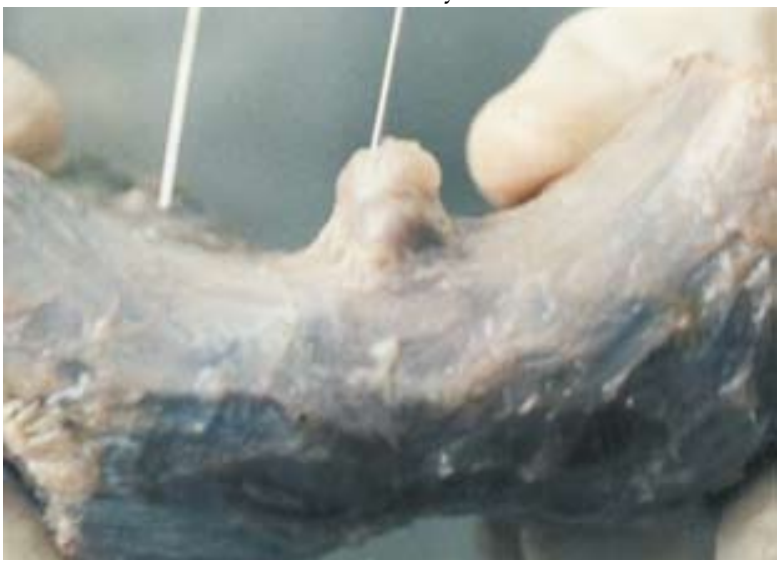

Figura 4. Vista superior de la cara posterior de la sínfisis púbica donde se ve en su parte media un reborde o cresta con orientación vertical.



protuberancias nodulares, se presume que puede tratarse de dos nodulaciones sinfisiarias en su cara posterior, a manera de pseudoquistes o nódulos perfectamente definidos, firmes, no secretantes y con paredes tenues y fibrosas que encierran una sustancia mucoide que es continúa con la cápsula articular del disco sinfisiario. Según Angelides ${ }^{18}$ y Gerstner, ${ }^{19}$ las nodulaciones más notorias se encuentran en las articulaciones de los miembros superiores, que consisten en quistes sinoviales en las articulaciones de libre movimiento, como las diartrosis carpianas.

En el caso de la sínfisis con presencia de cresta o reborde no se detectó comunicación directa con la 
cavidad del disco sinfisiario y se presume que pudo tratarse de una leve herniación.

En los dos casos citados no hay concordancia con la anatomía de la sínfisis púbica, pudiendo ser que los cadáveres evaluados correspondan a personas que por alguna razón desarrollaron esta variación o alteración anatómica, que bien puede ser de carácter patológico, pues ninguno de los cadáveres estudiados presentó muerte por trauma.

Los detalles del estudio de la anatomía macroscópica de la región sinfisiaria púbica y sus relaciones articulares correspondientes, nos permite proponer la existencia, poco corriente, de saculaciones no pediculadas que emergen desde la bolsa sinovial intraarticular a través del disco sinfisiario, ocupando la pared posterior de dicha articulación. Pudiera tratarse de quistes cartilaginosos subpúbicos, que fueron descritos inicialmente en 1996 y se han reportado en varios casos, como el de una mujer de 62 años que presentó cambios degenerativos extensos en la región anterior de la sínfisis púbica, compatibles con un quiste cartilaginoso subpúbico, ${ }^{20}$ los cuales son considerados lesiones raras y benignas. ${ }^{21}$

Aunque funcionalmente la articulación sinfisiaria púbica no es de tipo diartrósica, por su estructura sí podría ser catalogada de este tipo, debido a la presencia y apariencia de dichas nodulaciones, las que pudieron haber surgido espontáneamente o por desarrollo secundario a una lesión por trauma del disco interpúbico con la subsecuente perforación del tejido conectivo laxo, formándose finalmente dos sacos para el escape del líquido sinovial.

El análisis histológico se frustró debido al inadecuado procesamiento al que fue sometida la muestra, lo que impidió corroborar o descartar la presencia de células típicas de la cápsula articular en las paredes de las dos protuberancias en cuestión.

\section{CONCLUSIÓN}

Con respecto a las nodulaciones mencionadas, se concluye que puede tratarse de alteraciones patológicas articulares, tales como diastasis púbicas pero sin separación de los cuerpos del pubis.

\section{REFERENCIAS}

1. Geneser F. Histología. $1^{\mathrm{a}}$ ed. Buenos Aires: Editorial Médica Panamericana; 1988.

2. More KL, Dalley AF. Anatomía con orientación clínica. $5^{\mathrm{a}}$ ed. México: Editorial Médica Panamericana; 2007.

3. O’Rahilly R. Anatomía de Gardner. 5a ed. México: McGraw Hill Interamericana; 1989.

4. Lesson TS, Lesson RC. Histología. $4^{\mathrm{a}}$ ed. México: Nueva Editorial Interamericana S.A de C.V.; 1984.

5. Spalteholz W. Atlas de anatomía humana. $5^{\mathrm{a}} \mathrm{ed}$. Barcelona: Labor S.A; 1970.

6. Snell R. Anatomía clínica para estudiantes de medicina. $6^{a}$ ed. México: McGraw-Hill Interamericana; 2002.

7. Dauber W. Pocket atlas of human anatomy. $5^{\text {th }}$ Ed. New York: Thieme; 2007.

8. Pansky B. Review of gross anatomy. $6^{\text {th }}$ edition. New York: McGraw-Hill; 1996.

9. Testeut L, Latarjet A. Tratado de Anatomía Humana. $9^{a}$ Ed. Barcelona: Salvat Editores S.A.; 1954.

10. Carr BR, Bradshaw KD. Enfermedades del ovario y del aparato reproductor femenino. En: Kasper DL, Fauci AS, Longo DL, Braunwald E, Hauser SL, Jameson JL, editores. Principios de medicina interna. $16^{a}$ ed. México: Mc Graw-Hill Interamericana; 2006. p. 2418-31.

11. Guyton AC, Hall JE. Tratado de fisiología médica. $11^{\text {a }}$. ed. Madrid: Elsevier; 2006.

12. Ganong WF. Fisiología médica. 20ª ed. México: El manual moderno S.A de C.V.; 2005.

13. Botero UJ, Jubiz HA, Henao G. Obstetricia y Ginecología. 3ª Ed. Medellín: Carvajal S.A.; 1986.

14. Dunivan GC, Hickman AM, Connolly A. Severe separation of the pubic symphysis and prompt orthopedic surgical intervention. Obstet Gynecol 2009;114:473-5.

15. Chang JL, Wu V. External fixation of pubic symphysis diastasis from postpartum trauma. Orthopedics 2008;31:493.

16. Salter RB. Trastornos y lesiones del sistema músculo esquelético. $1^{\text {a }}$ ed. Barcelona: Salvat Editores S.A.; 1971.

17. Robertson BA, Barker PJ, Fahrer M, Schache AG. The anatomy of the pubic region revisited: implications for the pathogenesis and clinical management of chronic groin pain in athletes. Sports Med 2009;39:225-34. 
18. Angelides AC, Wallace FP. The dorsal ganglion of the wrist: its pathogenesis, gross and microscopic anatomy, and surgical treatment. J Hand Surg Am 1976;1:228-35.

19. Gerstner J. Gangliones. Colomb Med 1997;28:226.
20. Judson PL, Ivy JJ, Zwolak P, Manivel JC, Clohisy DR. A suspicious vulvar mass diagnosed as a subpubic cartilaginous cyst. Arch Gynecol Obstet 2009;280:107-9.

21. Ergun T, Lakadamyali H, Aydin O. Subpubic cartilaginous cyst: incidental finding detected by abdominopelvic computed tomography. Radiat Med 2008;26:46-9.

Conflicto de intereses: ninguno declarado. 